\title{
Non-alcoholic Steatohepatitis: Comparison of Intestinal Microbiota between Different Metabolic Profiles. A Pilot Study
}

\author{
Juliano Machado de Oliveira ${ }^{1}$, Fábio Heleno de Lima Pace ${ }^{1}$, Fabiana de Faria Ghetti ${ }^{1}$, Kátia Valéria Bastos Dias \\ Barbosa $^{1}$, Dioneia Evangelista Cesar ${ }^{2}$, Julio Maria Fonseca Chebli ${ }^{1}$, Lincoln Eduardo Villela Vieira de Castro Ferreira ${ }^{1}$
}

1) University Hospital and

School of Medicine, Federal University of Juiz de Fora 2) Biology Department, Federal University of Juiz de Fora, Brazil
Received: 26.10 .2019 Accepted: 18.05 .2020

\section{ABSTRACT}

Background \& Aims: Non-alcoholic steatohepatitis (NASH) has multifactorial etiopathogenesis, and intestinal microbiota is co-responsible in this process. The aim of this study was to evaluate the intestinal microbiota in NASH patients with different metabolic profiles.

Methods: Patients with biopsy-proven NASH were evaluated. Subjects were divided into two groups according to their metabolic profile, with or without metabolic syndrome (MS). Their characteristics in relation to liver disease and intestinal microbiota were analyzed. To evaluate the microbiota, breath tests to investigate small intestinal bacterial overgrowth (SIBO) and fecal microbiota analysis by fluorescence in situ hybridization (FISH) were performed.

Results: There was a high prevalence of SIBO in both groups, with no significant difference between them. Breathing tests were positive in $43.8 \%$ of patients with MS and $50 \%$ of those without MS. There was a significant difference regarding the quantification of Verrucomicrobiales, less abundant in patients with NASH without MS. Its lower concentration also correlated with higher serum ferritin levels and higher hepatocyte ballooning. This order of bacteria, through its representative in human microbiota, Akkermansia muciniphila, is associated with mucosal protection and metabolic processes with liver aggression.

Conclusions: Our results suggested that lower Verrucomicrobiales concentration is associated with higher inflammatory activity in patients with NASH without MS, where the disease etiopathogenesis does not have its classic metabolic substrate.

Key words: non-alcoholic fatty liver disease - intestinal microbiota - metabolic syndrome - steatohepatitis - dysbiosis.

Abbreviations: ALT: alanine aminotransferase; AST: aspartate aminotransferase; BMI: body mass index; GGT: gamma glutamyltransferase; HDL: high density lipoprotein; HOMA IR: homeostasis model assessment of insulin resistance; LDL: low density lipoprotein; MS: metabolic syndrome; NAFLD: nonalcoholic fatty liver disease; NASH: nonalcoholic steatohepatitis; NAS: NAFLD activity score; NFS: NAFLD fibrosis score; SIBO: small intestinal bacterial overgrowth.

\section{INTRODUCTION}

Currently, nonalcoholic fatty liver disease (NAFLD) is the most common chronic liver disease in the world population. Its average prevalence is $20 \%$ but varies between 6 and 35\% [1]. The spectrum of presentation is broad, ranging from pure steatosis to non-alcoholic steatohepatitis (NASH) presenting inflammation, ballooning, and variable degrees of fibrosis affecting 2 to $3 \%$ of the general population. NASH has evolutionary potential for liver cirrhosis and hepatocellular carcinoma $[2,3]$. However, its evolution is heterogeneous and depends on multiple factors such as age, body mass index (BMI), and insulin resistance with or without diabetes [4]. The main pathophysiological mechanism of NAFLD is insulin resistance, frequently identified in patients with metabolic syndrome (MS), thus considered the hepatic manifestation of this disorder. However, a significant proportion of patients develop NAFLD in the absence of a metabolic syndrome. Recently, qualitative and quantitative changes in the intestinal microbiota have been implicated as a possible mechanism associated with NAFLD.

The mechanisms by which changes in the intestinal microbiota lead to fat deposition in the liver parenchyma 
and potential liver injury are still speculative. Possible factors include altered energy balance associated with MS and NAFLD $[5,6]$, increased bioproducts such as lipopolysaccharides (LPS) [7-9] and alcohol [10-12] generated by intestinal bacteria and increased intestinal permeability secondary to small intestinal bacterial overgrowth (SIBO) [13-15]. Nevertheless, not only quantitative changes in the intestinal microbiota are implicated in the pathogenesis of NAFLD. Recent studies have shown a protective role of some intestinal bacteria with respect to metabolic diseases and NAFLD [16]. However, there is still no clarity about the causal relationship between changes in gut microbiota and NAFLD. Recently, Ghetti et al. [17] evaluated the role of diet in patients with NASH. A beneficial effect was observed on the intestinal microbiota profile and on metabolic and hepatic parameters in the group that received nutritional intervention in relation to the control group [17].

The way dysbiosis interferes with this process is still obscure and probably diverse. Which microorganisms play a potential role in the genesis and progression of NASH must be better defined. Another important question is whether microbiota can play a role in NASH development even in individuals without MS. In this study, we aimed to identify the characteristics of the intestinal microbiota in patients with NASH according to their metabolic condition.

\section{METHODS}

\section{Study design}

We conducted a cross-sectional observational study with prospective inclusion of patients recruited by convenience, at the NAFLD outpatient clinic of the Gastroenterology Service, University Hospital, the Federal University of Juiz de Fora (UFJF), Minas Gerais, Brazil. All subjects signed an informed consent form before inclusion in the study. The study was approved by the UFJF Ethics Committee under number 10994513.0.0000.5133.

\section{Patient selection}

Patients older than 18 years of age with suspected NAFLD through findings suggestive of steatosis on imaging examination without other identifiable liver diseases such as alcoholic liver disease (ethanol consumption above $20 \mathrm{~g} / \mathrm{day}$ for women and $40 \mathrm{~g} /$ day for men), drug-induced liver disease, viral hepatitis B or C, autoimmune liver disease, Wilson's disease and hemochromatosis, were selected. They were followed for at least six months, with lifestyle change medical advice that included physical activity and diet according to their individualities. Patients with maintained elevated aminotransferases above $1.5 \mathrm{x}$ the upper limit of normality were invited to participate in the study. Those who agreed were submitted to a liver biopsy according to the standard workup of our service. Subjects were included in the study if they had histological confirmation of $\mathrm{NASH}$ without markers of other liver diseases. Individuals infected with the human immunodeficiency virus (HIV) were excluded, as well as diabetics with chronic or decompensated complications and with decompensated liver disease (ChildPugh B or C) due to the potential role of these conditions on the intestinal microbiota. After inclusion in the study, the patients were divided into two groups: with MS (MS) and without MS (non MS) (Fig.1). The diagnosis of MS was established according to the National Cholesterol Education Program (NCEP) Expert Panel on Detection, Evaluation, and Treatment of High Blood Cholesterol in Adults (Adult Treatment Panel III) - NCEP-ATP III [18].

\section{Clinical and laboratory evaluation}

Blood pressure levels and anthropometric data were recorded, including weight, height, BMI, and abdominal waist. The levels of alanine aminotransferase (ALT), aspartate aminotransferase (AST), gamma glutamyltransferase (GGT), bilirubin, albumin, prothrombin time and activity, glucose, insulin, total cholesterol, high density lipoprotein (HDL), low density lipoprotein (LDL), and triglycerides were determined. The Homeostasis Model Assessment of Insulin Resistance (HOMA IR) was calculated from fasting glucose and insulin [19]. From clinical and laboratory parameters, noninvasive fibrosis scores (NAFLD Fibrosis Score - NFS [20] and FIB-4) were calculated [21]. In addition, tests were performed to rule out other possible liver diseases: $\mathrm{HBsAg}$, anti-HCV, antinuclear antibody (ANA), anti-smooth muscle antibody (SMA), antimitochondrial antibody (AMA), ferritin, transferrin saturation, ceruloplasmin, and anti-HIV.

\section{Histological analysis}

The presence of steatosis above 5\%, ballooning, and inflammatory infiltrate on histological analysis was considered the definitive diagnosis of NASH. Histological activity and degree of fibrosis were graded according to the NAFLD activity score (NAS) score [22].

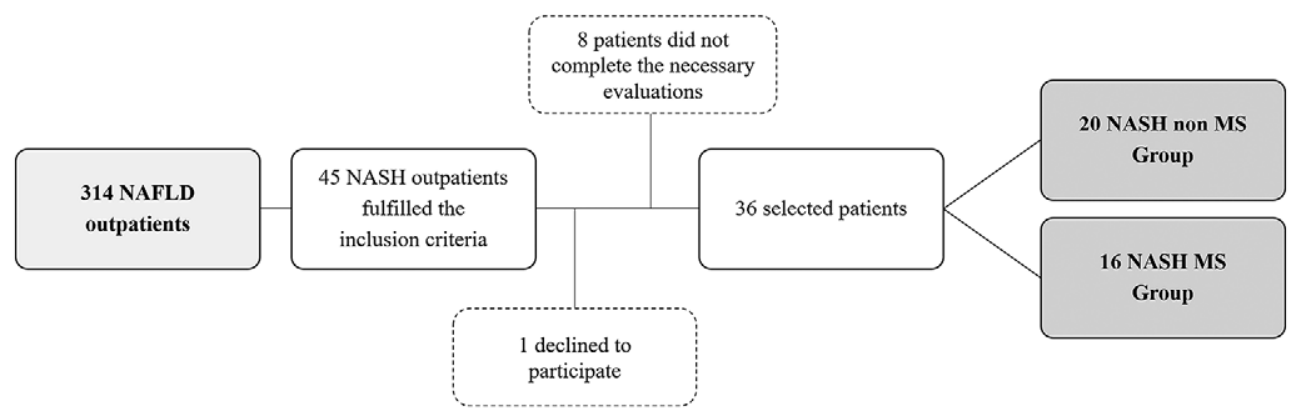

Fig. 1. Recruitment flow chart. NAFLD, nonalcoholic fatty liver disease; NASH, non-alcoholic fatty liver disease; MS, group of patients with steatohepatitis with metabolic syndrome; non MS, group of patients with steatohepatitis without metabolic syndrome. 


\section{SIBO assessment}

For SIBO evaluation, all subjects underwent the breath test. The tests were performed on the QuinTron BreathTracker ${ }^{\text {Tix }}$ Digital MicroLyzer apparatus, where expired hydrogen $\left(\mathrm{H}_{2}\right)$ and methane $\left(\mathrm{CH}_{4}\right)$ elimination were tested after the ingestion of $15 \mathrm{ml}$ of lactulose $667 \mathrm{mg} / \mathrm{ml}$. The apparatus is an analyzer that measures $\mathrm{H}_{2}$ and $\mathrm{CH}_{4}$ of the gas sample in parts per million (ppm).

On the eve of the exam, subjects were instructed to follow a non-fermentative diet. They were not on antibiotics, probiotics, laxatives, or proton pump inhibitors in the preceding week. The exam was done after a 10-hour fast. Each subject collected expired alveolar air in specific collection bags prior to carbohydrate ingestion (time 0 ) and after use of the test substance (lactulose) at times 15‘, 30`, 45،, 60`, 90`, and 120`. The collected samples were immediately analyzed.

Data obtained from the analysis of the samples were evaluated in curves and elevations of $\mathrm{H}_{2}$ above $20 \mathrm{ppm}$ or $\mathrm{CH}_{4}$ above 10 ppm from basal dosage up to $90^{`}$ were considered positive for SIBO [23].

\section{Fecal microbiota characterization}

Stool samples were collected in sterile vials and kept refrigerated for up to 24 hours. They were referred to the Laboratory of Ecology and Molecular Biology of Microorganisms of UFJF. The feces were processed for microbiological analysis by fluorescent in situ hybridization (FISH) (Supplementary Table I). They were fixed with paraformaldehyde ( $2 \%$ final concentration), and a $0.01 \%$ Tween solution was added to each sample. Then the samples were sonicated (Vibra Cell VCX 130PB, Sonics \& Materials ${ }^{\circledR}$ ) three times (range $110.7 \mu \mathrm{m}$ for 60 seconds). After sonication, they were centrifuged at $500 \mathrm{~g}$ for five minutes. The supernatant was removed, and the remaining contents were washed twice with ultrapure water. The three supernatant fractions were placed in one vial and shaken vigorously. Aliquots of each sample were diluted $(100 \mathrm{x})$, filtered through polycarbonate filters (Nuclepore ${ }^{\varpi}-0.2 \mu \mathrm{m}$ ) and stored in a refrigerator until the hybridization process. Subsequently, the samples were submitted to the FISH protocol [24] to identify and quantify the microbial groups of interest [17]. A negative control probe (5'CCTAGTAGACGCCGTCGAC-3'), which has no specificity for any bacterial group, was also used to evaluate hybridization efficiency. Probes were labeled with fluorochrome Cy3. The density of microorganisms ( $\times 10^{8}$ cells $\mathrm{g}^{-1}$ ) was determined by direct counting at 100x magnification using an epifluorescence microscope (Olympus ${ }^{\oplus}$ BX-60) equipped with the U-N41007, U-MWU2, U-MWB2, and U-MWG2 optical filter set.

\section{Statistical analysis}

Statistical analysis was performed using SPSS $^{\circledR}$ software (version 16.0 for Windows., SPSS Inc., United States of America). Parametric and nonparametric tests were used according to the normality (Shapiro-Wilk) and homogeneity of variance (Levene) tests. Continuous variables were represented as mean and standard deviation. Categorical variables were expressed as relative and absolute frequency (n). Spearman's correlation was used to evaluate bivariate relationships between variables. The $t$-test for independent samples or Mann-Whitney $U$ test was used to compare continuous variables between groups.
Categorical variables were compared using the Chi-square test or Fisher's exact test (between groups). A significance level of $0.05(\alpha=5 \%)$ was adopted. Descriptive levels equal to or lower than this value were considered significant.

\section{RESULTS}

\section{Patients}

During the study period, 314 patients were followed. Forty patients with biopsy indication according to the adopted criteria agreed to participate in the study. Thirty-six patients with histological confirmation of NASH were included. Two patients selected for biopsy did not have histological criteria for $\mathrm{NASH}$ and two patients did not complete the analyses required for the study. The main clinical, laboratory, and histological characteristics of the groups are shown in Table I. From the total of selected patients, 16 met criteria for MS. The mean age was similar between groups, and female and male gender prevailed in the MS and non MS groups, respectively.

As expected, there was significant difference between groups regarding MS criteria, besides BMI and HOMA IR. In contrast, there was no difference in liver disease presentation in any of the studied parameters, such as aminotransferase level, serum ferritin, noninvasive fibrosis markers (NAFLD Fibrosis Score - NFS and FIB-4), and histological characteristics. Histological analysis confirmed the diagnosis of NASH in all patients, without a marker of another liver disease. Most patients had mild fibrosis with histological stage $\mathrm{F}<2$ in $85 \%$ of the non MS group and $87.5 \%$ of the MS group. There was also no difference in relation to the other histological parameters, such as degree of steatosis, ballooning, inflammation and the activity score (NAS).

The evaluation of SIBO determined by both $\mathrm{H}_{2}$ and $\mathrm{CH}_{4}$ was frequent in all samples regardless of the metabolic profile (Fig. 2a). There was also no significant difference in the prevalence of bacteria producing $\mathrm{H}_{2}$ or $\mathrm{CH}_{4}$ between the two groups. SIBO was positive in $43.8 \%\left(25 \% \mathrm{H}_{2}\right.$ and $18.8 \%$ for both $\mathrm{H}_{2}$ and $\left.\mathrm{CH}_{4}\right)$ of MS subjects and $50 \%\left(30 \% \mathrm{H}_{2}, 15 \% \mathrm{CH}_{4}\right.$, and $5 \%$ for both $\mathrm{H}_{2}$ and $\mathrm{CH}_{4}$ ) of those non MS (Fig. 2b).

Regarding qualitative analysis of the microbiota, the distribution of the order Verrucomicrobiales (from the phylum Verrucomicrobia) showed a significant difference $(p=0.01)$ between the groups, being lower in those without MS (Fig. 3a).

In the analysis of the entire sample, Verrucomicrobiales had inverse correlation with parameters related to liver inflammation. Individuals with a higher concentration of Verrucomicrobiales had lower hepatic ballooning ( $\mathrm{R}-0.37$ / $\mathrm{p}$ 0.02 ) and lower serum ferritin level (R -0.34 / p 0.04) (Fig. 4). All other microbial groups studied had similar distribution of concentrations regardless of metabolic condition (Table II). However, the study of the Ruminococcaceae family (from the Firmicutes phylum) showed a tendency $(p=0.058)$ for concentration differences, being less abundant in the group without MS (Fig. 3b).

\section{DISCUSSION}

Our study assessed the differences between NASH patients with or without MS. Among all the parameters evaluated in the 
Table I. Clinical, laboratory and histological characteristics of the patients

\begin{tabular}{|c|c|c|c|c|c|c|c|}
\hline & \multicolumn{3}{|c|}{ non MS } & \multicolumn{3}{|c|}{ MS } & \multirow[t]{2}{*}{$\mathrm{p}$} \\
\hline & $\%$ & $\mathrm{n}=20$ & Mean \pm SD & $\%$ & $\mathrm{n}=16$ & Mean \pm SD & \\
\hline Age, years & & & $47.5 \pm 10.5$ & & & $49.5 \pm 9.8$ & 0.552 \\
\hline Gender (female) & 27.8 & 5 & & 72.2 & 13 & & 0.001 \\
\hline Obesity & 45 & 9 & & 75 & 12 & & 0.020 \\
\hline High WC & 25 & 5 & & 81.2 & 13 & & 0.001 \\
\hline High Glucose & 10 & 2 & & 43.8 & 7 & & 0.050 \\
\hline Low HDL & 20 & 4 & & 93.8 & 15 & & $<0.001$ \\
\hline High TG & 50 & 10 & & 93.8 & 15 & & 0.009 \\
\hline Hypertension & 10 & 2 & & 81.3 & 13 & & $<0.001$ \\
\hline MS, no of criteria & & & $1.15 \pm 0.9$ & & & $3.9 \pm 0.7$ & $<0.001$ \\
\hline BMI Kg/m² & & & $29.9 \pm 3.46$ & & & $33.8 \pm 5.8$ & 0.020 \\
\hline HOMA- IR & & & $2.96 \pm 1.04$ & & & $5.16 \pm 2.54$ & 0.004 \\
\hline AST, U/L & & & $47.5 \pm 25.9$ & & & $47.1 \pm 25.8$ & 0.080 \\
\hline $\mathrm{ALT}, \mathrm{U} / \mathrm{L}$ & & & $57.5 \pm 26.5$ & & & $60.7 \pm 38.3$ & 0.514 \\
\hline GGT, U/L & & & $77.6 \pm 59.9$ & & & $92.4 \pm 81.8$ & 0.368 \\
\hline FTI, ng/mL & & & $313 \pm 185.7$ & & & $241 \pm 173.8$ & 0.699 \\
\hline $\operatorname{PLT}\left(\mathrm{x} 10^{9} / \mathrm{L}\right)$ & & & $212 \pm 49$ & & & $235 \pm 71$ & 0.159 \\
\hline NFS & & & $1.07 \pm 1.43$ & & & $1.33 \pm 1.52$ & 0.691 \\
\hline FIB-4 & & & $1.6 \pm 1.08$ & & & $1.53 \pm 1.11$ & 0.874 \\
\hline \multicolumn{8}{|l|}{ Biopsy } \\
\hline Steatosis & & & $2.15 \pm 0.67$ & & & $2.5 \pm 0.63$ & 0.662 \\
\hline Lob Inf & & & $1.1 \pm 0.64$ & & & $1.13 \pm 0.5$ & 0.384 \\
\hline Ballooning & & & $1.55 \pm 0.68$ & & & $1.63 \pm 0.5$ & 0.139 \\
\hline NAS & & & $4.8 \pm 1.05$ & & & $5.19 \pm 0.91$ & 0.499 \\
\hline Fibrosis $\geq 2$ & 15 & 3 & & 12.5 & 2 & & 0.829 \\
\hline BT-SIBO & 50 & 10 & & 43.8 & 7 & & 0.629 \\
\hline
\end{tabular}

Data presented as mean and standard deviation (SD) or as a percentage of prevalence in the group and number of individuals (n). Student's t- test for numeric variables. Chi-square for categorical.

Abbreviations: MS: group of patients with steatohepatitis with metabolic syndrome; non MS: group of patients with steatohepatitis without metabolic syndrome; MS - no criteria: number of metabolic syndrome criteria; WC: waist circumference; HDL: high density lipoprotein; LDL: low density lipoprotein; TG: triglycerides; BMI: body mass index; HOMAR-IR: homeostasis model assessment of insulin resistance; AST: aspartate aminotransferase; ALT: alanine aminotransferase; GGT: gamma glutamyltransferase; FTI: ferritin; PLT: platelets; NFS: NAFLD fibrosis score; FIB-4: fibrosis-4 score; Lob Inf: lobular inflammation; NAS: NAFLD activity score; BT-SIBO: positive breath test for small intestinal bacterial overgrowth; ns: not significant.

differentiation of groups, the only significant one was related to intestinal microbiota.

Hepatic evaluation was similar between groups in this study, with no metabolic influence over liver disease progression. Both groups were also similar in relation to all other histological, laboratory, and noninvasive markers of hepatic evaluation. However, it is noteworthy that, according to the exclusion criteria adopted, we excluded patients with advanced liver cirrhosis and diabetes mellitus with signs of complication due to the potential secondary influence of these conditions on the intestinal microbiota. The exclusion of these patients may have influenced the severity profile of liver disease in our sample, especially in the MS group. In the global $\mathrm{NASH}$ population, it is expected that the higher the severity of metabolic disease, the greater the risk of progression to advanced stages over time $[4,25,26]$. However, this exclusion criterion did not impact the result of the study, which aimed to identify the association of intestinal microbiota with NASH and not with the state of advanced liver disease.

There was a higher frequency of female gender among MS patients and male among those without MS. This may be due to selection bias in a referral center with a predominance of obese women and men with difficult etiologic diagnosis of elevated liver enzymes without evidence of metabolic changes or other liver diseases.

To evaluate the intestinal microbiota, we investigated the presence of SIBO. There was a high prevalence of this condition among patients, but without difference between the groups with or without MS. The test used to detect SIBO was considered positive in the presence of an increase either in $\mathrm{H}_{2}$ or $\mathrm{CH}_{4}$, optimizing the sensitivity of the method. In the analysis of the density of groups of microorganisms of interest for this study of fecal microbiota, we observed a difference in two microorganisms. There was a statistical tendency of 

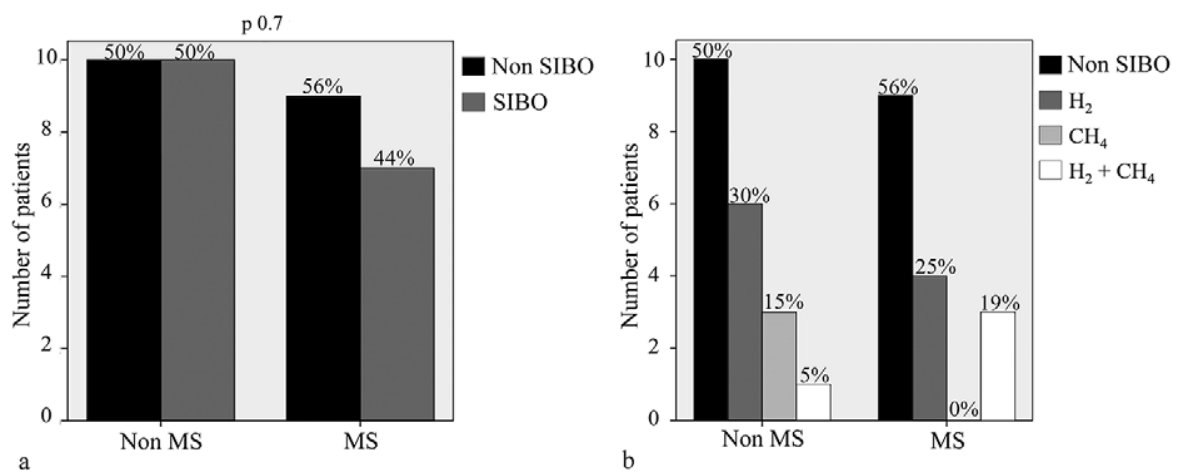

Fig. 2. Breath test for SIBO according to metabolic status (with or without MS). MS, group of patients with steatohepatitis with metabolic syndrome; non MS, group of patients with steatohepatitis without metabolic syndrome; non SIBO, breath test negative for SIBO; SIBO, breath test positive for SIBO; $\mathrm{H}_{2}$, breath test positive for hydrogen; $\mathrm{CH}_{4}$, breath test positive for methane; $\mathrm{H}_{2}+\mathrm{CH}_{4}$, breath test positive for both.

lower concentration of the Ruminococcaceae family in the group without MS. This data is in line with other studies that evaluated NASH in thin individuals [27]. This family has been related to a higher presence of advanced fibrosis in patients with NASH [28]. This relationship was not observed in our group with a low prevalence of advanced fibrosis due to the exclusion criterion adopted.

The Verrucomicrobiales family was also less populous in the group without MS, but with a significant difference. In 2004, Akkermansia muciniphila [29], an anaerobic gram negative bacteria representing Verrucomicrobiales in the human intestinal microbiota [30] was isolated. This bacterium degrades mucin as a source of carbon and nitrogen and
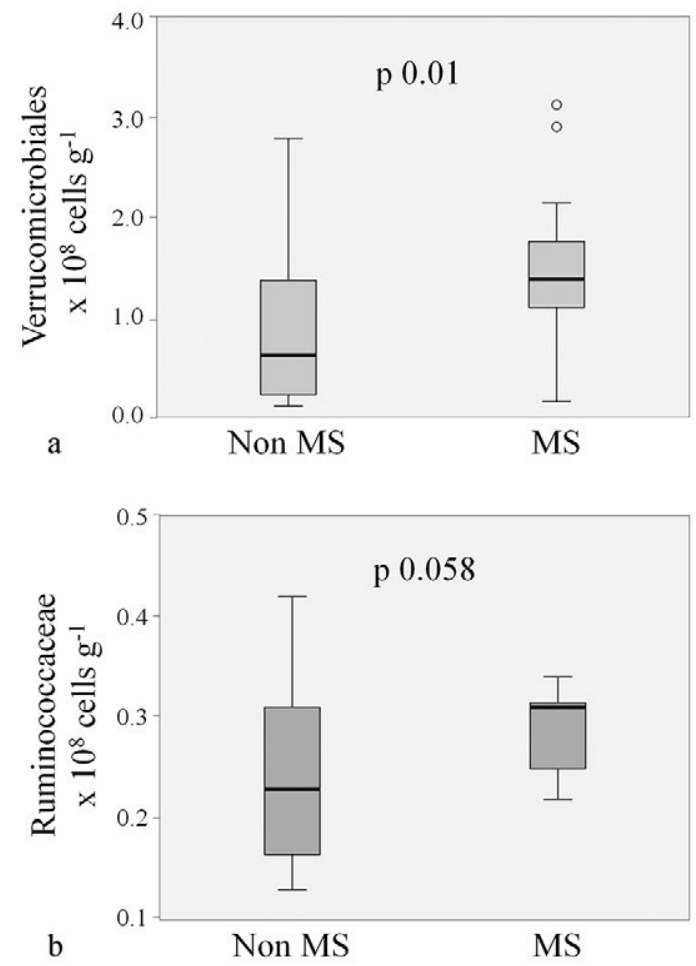

Fig. 3. Distribution of Verrucomicrobiales (a) and Ruminococcaceae (b) according to metabolic status (with or without MS). stimulates turnover of the intestinal barrier. In addition, it generates oligosaccharides and short chain fatty acids that stimulate mucosal barrier protective bacteria, the immune response, and leads to gene stimulation of the peroxisome proliferator-activated receptors (PPARa)-associated metabolic pathways [31]. It has been described throughout the digestive tract, but predominantly in the small intestine and colon [32]. Since its identification, several studies have shown its protective role for the mucosal barrier, finding its abundance in healthy intestines and a decrease in conditions accompanied by intestinal injury and inflammation, such as appendicitis and inflammatory bowel diseases $[33,34]$. Akkermansia muciniphila has also been associated with prominent
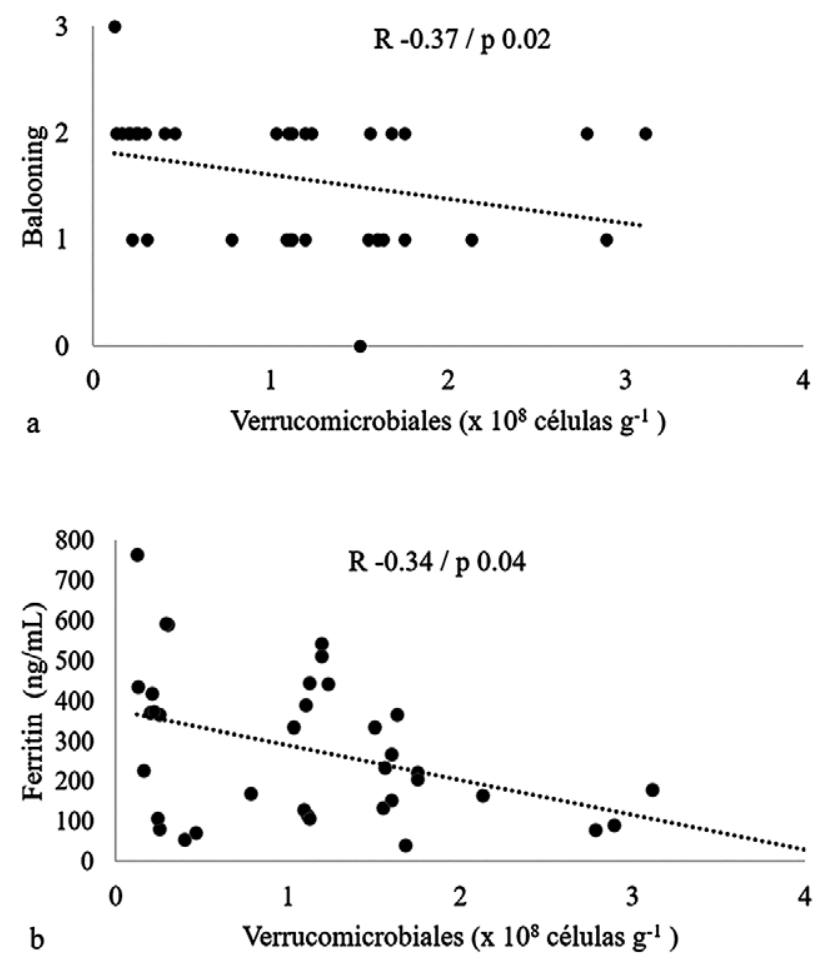

Fig. 4. Inverse correlation between the concentration of Verrucomicrobiales and hepatocyte ballooning (a) and serum ferritin (b). 


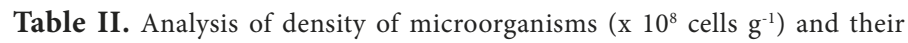
distribution according to metabolic status

\begin{tabular}{llll}
\hline Microbial Group & non MS, mean $( \pm$ SD) & MS, mean $( \pm$ SD) & $\mathrm{p}$ \\
\hline Bifidobacterium spp. & $0.22(0.098)$ & $0.21(0.095)$ & 0.937 \\
Lactobacillus & $0.24(0.108)$ & $0.20(0.076)$ & 0.305 \\
Enterococcus & $0.28(0.084)$ & $0.28(0.074)$ & 1.000 \\
Escherichia coli & $0.30(0.098)$ & $0.29(0.135)$ & 0.386 \\
Bacteroides & $0.30(0.100)$ & $0.35(0.105)$ & 0.262 \\
Prevotella & $0.43(0.089)$ & $0.45(0.133)$ & 0.937 \\
Streptococcus & $0.28(0.072)$ & $0.29(0.057)$ & 0.694 \\
Ruminococcaceae & $0.24(0.087)$ & $0.29(0.042)$ & 0.058 \\
Aeromonas & $0.27(0.080)$ & $0.27(0.051)$ & 0.671 \\
Clostridium & $0.27(0.116)$ & $0.31(0.088)$ & 0.211 \\
Bacteroidetes & $2.49(1.466)$ & $2.77(1.660)$ & 0.560 \\
Firmicutes & $1.35(0.633)$ & $1.35(0.806)$ & 0.937 \\
Actinobacteria & $1.39(0.775)$ & $1.24(0.788)$ & 0.560 \\
Acidobacteria & $1.07(0.708)$ & $1.33(0.625)$ & 0.276 \\
Archaea & $0.93(0.605)$ & $0.88(0.529)$ & 0.888 \\
Verrucomicrobiales & $0.84(0.724)$ & $1.44(0.823)$ & 0.016 \\
\hline MS: group of patients with steatohepatitis with metabolic syndrome; non MS: group \\
of patients with steatohepatitis without metabolic syndrome.
\end{tabular}

influence on the metabolic profile. There is evidence of a negative relationship between Akkermansia muciniphila and interferon $\gamma$, insulin resistance-related cytokine [35], and other inflammatory cytokines related to metabolic disorders [36]. It influences the relationship between intestinal microbiota and host, especially in patients with obesity and type 2 diabetes [37].

In a study by our team that evaluated the role of diet in patients with $\mathrm{NASH}$, the group that received dietary intervention increased the overall concentration of bacteria in the fecal microbiota analysis, while in the control group, a reduction in Verrucomicrobiales concentration was observed [17]. Decreasing Verrucomicrobiales, due to its beneficial role on the mucosal barrier [31], may lead to increased intestinal permeability and consequent participation in liver aggression [15]. It is also worth mentioning the epigenetic influence of the eating pattern in patients with NAFLD [38]. However, the potential role of microbiota in this gene expression needs further investigation.

In the present study, a lower concentration of Verrucomicrobiales was associated with increased hepatocyte ballooning and higher serum ferritin levels, markers of NASH inflammatory activity. Interestingly, this order of bacteria was less abundant in the NASH group without MS. This group has NASH without the usual substrate of metabolic changes. In these cases, the etiopathogenesis of liver injury is still a matter of controversy and the possible role of intestinal microbiota is of great interest.

The dietary habits of the individuals in this study were evaluated in relation to the daily intake of energy and nutrients (carbohydrates, fibers, lipids, saturated and unsaturated fats, cholesterol and proteins). There was no impact on the concentration of Verrucomicrobiales regardless of the dietary profile (unpublished data).

Although not attributable to cause and effect, our results suggest that the lower abundance of Verrucomicrobiales increases the risk of inflammatory activity in NASH patients, especially in NASH patients without MS. In this group without the necessary criteria for the diagnosis of MS, almost half of the patients were obese and had, on average, a criterion of MS. However, many patients without MS are of difficult therapeutic adequacy, given that the measures for metabolic control that underlie NASH management are not always justified for their profile. The role of the intestinal microbiota, in particular the lower concentration of Verrucomicrobiales, makes room for further studies. Some experiments have been conducted, analyzing the therapeutic role of A. muciniphila as a probiotic [39-41]. Although in a short period of dietary intervention, Ghetti et al. [17] demonstrated that diet was able to maintain Verrucomicrobiales levels. Further studies with long-term dietary intervention are required to demonstrate its role in general microbiota modulation, with better control of the NASH inflammatory process, especially for patients without MS.

\section{CONCLUSIONS}

Our results suggested that lower Verrucomicrobiales concentration is associated with higher inflammatory activity in patients with NASH without MS. This data suggests its protective role with a possible association with disease progression in those individuals with NASH without MS, where Verrucomicrobiales was less populous. Studies must be conducted that evaluate the role of specific bacteria such as Akkermansia muciniphila and the interaction with the entire intestinal ecosystem, as well as the best intervention for its modulation.

Conflicts of interest: None to declare.

Authors' contributions: J.M.O. conceived and designed the study. J.M.O and F.F.G. collected data and performed the respiratory tests. 
F.H.L.P. and K.V.B.D.B. performed the statistical analysis. F.F.G. and D.E.C. analyzed the fecal microbiota. J.M.F.C. and L.E.V.V.C.F. analyzed the data and drafted the manuscript. All authors critically revised the manuscript, approved the final version to be published, and agree to be accountable for all aspects of the work.

Acknowledgements: The authors gratefully acknowledge the support by the Brazilian Government Organization (Fundacao de Amparo a Pesquisa do Estado de Minas Gerais-FAPEMIG; APQ 01522-15).

Supplementary material: To access the supplementary material visit the online version of the J Gastrointestin Liver Dis at http://dx.doi. org/10.15403/jgld-497

\section{REFERENCES}

1. Vernon G, Baranova A, Younossi ZM. Systematic review: the epidemiology and natural history of non-alcoholic fatty liver disease and non-alcoholic steatohepatitis in adults. Aliment Pharmacol Ther 2011;34:274-285. doi:10.1111/j.1365-2036.2011.04724.x

2. Browning JD, Szczepaniak LS, Dobbins R, et al. Prevalence of hepatic steatosis in an urban population in the United States: Impact of ethnicity. Hepatology 2004;40:1387-1395. doi:10.1002/hep.20466

3. Clark JM, Brancati FL, Diehl AM. The prevalence and etiology of elevated aminotransferase levels in the United States. Am J Gastroenterol 2003;98:960-967.

4. Ekstedt M, Franzén LE, Mathiesen UL, et al. Long-term follow-up of patients with NAFLD and elevated liver enzymes. Hepatology 2006;44:865-873. doi:10.1002/hep.21327

5. Le Roy $\mathrm{T}$, Llopis $\mathrm{M}$, Lepage $\mathrm{P}$, et al. Intestinal microbiota determines development of non-alcoholic fatty liver disease in mice. Gut 2012;62:1787-1794. doi:10.1136/gutjnl-2012-303816

6. de Faria Ghetti F, Oliveira DG, Oliveira JM, de Castro Ferreira LEVV, Cesar DE, Moreira APB. Influence of gut microbiota on the development and progression of nonalcoholic steatohepatitis. Eur J Nutr 2018;57:861-876. doi:10.1007/s00394-017-1524-X

7. Machado MV, Cortez-Pinto H. Gut microbiota and nonalcoholic fatty liver disease. Ann Hepatol 2012;11:440-449. doi:10.1016/s16652681(19)31457-7

8. Ghoshal S, Witta J, Zhong J, de Villiers W, Eckhardt E. Chylomicrons promote intestinal absorption of lipopolysaccharides. J Lipid Res 2009;50:90-97. doi:10.1194/jlr.m800156-jlr200

9. Laugerette F, Vors C, Peretti N, Michalski MC. Complex links between dietary lipids, endogenous endotoxins and metabolic inflammation. Biochimie 2011;93:39-45. doi:10.1016/j. biochi.2010.04.016

10. Cope K, Risby T, Diehl AM. Increased gastrointestinal ethanol production in obese mice: Implications for fatty liver disease pathogenesis. Gastroenterology 2000;119:1340-1347. doi:10.1053/ gast.2000.19267

11. Nair S, Cope K, Risby TH, Diehl AM. Obesity and female gender increase breath ethanol concentration: potential implications for the pathogenesis of nonalcoholic steatohepatitis. Am J Gastroenterol 2001;96:1200-1204. doi:10.1111/j.1572-0241.2001.03702.x

12. Zhu L, Baker SS, Gill C, et al. Characterization of gut microbiomes in nonalcoholic steatohepatitis (NASH) patients: A connection between endogenous alcohol and NASH. Hepatology 2013;57:601-609. doi:10.1002/hep.26093
13. Wigg AJ, Roberts-Thomson IC, Dymock RB, McCarthy PJ, Grose $\mathrm{RH}$, Cummins AG. The role of small intestinal bacterial overgrowth, intestinal permeability, endotoxaemia, and tumor necrosis factor alpha in the pathogenesis of non-alcoholic steatohepatitis. Gut 2001;48:206211. doi:10.1136/gut.48.2.206

14. Bures J, Cyrany J, Kohoutova D, et al. Small intestinal bacterial overgrowth syndrome. World J Gastroenterol 2010;16:2978-2990. doi:10.3748/wjg.v16.i24.2978

15. Miele L, Valenza V, La Torre G, et al. Increased intestinal permeability and tight junction alterations in nonalcoholic fatty liver disease. Hepatology 2009;49:1877-1887. doi:10.1002/hep.22848

16. Özkul C, Yalınay M, Karakan T, Yllmaz G. Determination of certain bacterial groups in gut microbiota and endotoxin levels in patients with nonalcoholic steatohepatitis. Turk J Gastroenterol 2017;28:361-369. doi:10.5152/tjg.2017.17033

17. De Faria Ghetti F, De Oliveira DG, Oliveira JM, et al. Effects of Dietary Intervention on Gut Microbiota and Metabolic-Nutritional Profile of Outpatients with Non-Alcoholic Steatohepatitis: a Randomized Clinical Trial. J Gastrointestin Liver Dis 2019;28:279-287. doi:10.15403/jgld-197

18. Expert Panel on Detection, Evaluation, and Treatment of High Blood Cholesterol in Adults. Executive Summary of The Third Report of The National Cholesterol Education Program (NCEP) Expert Panel on Detection, Evaluation, And Treatment of High Blood Cholesterol In Adults (Adult Treatment Panel III). JAMA 2001;285:2486-2497. doi:10.1001/jama.285.19.2486

20. Matthews DR, Hosker JP, Rudenski AS, Naylor BA, Treacher DF, Turner RC. Homeostasis model assessment: insulin resistance and $\beta$-cell function from fasting plasma glucose and insulin concentrations in man. Diabetologia 1985;28:412-419. doi:10.1007/bf00280883

21. Angulo P, Hui JM, Marchesini G, et al. The NAFLD fibrosis score: A noninvasive system that identifies liver fibrosis in patients with NAFLD. Hepatology 2007;45:846-854. doi:10.1002/hep.21496

22. Sun W, Cui H, Li N, et al. Comparison of FIB-4 index, NAFLD fibrosis score and BARD score for prediction of advanced fibrosis in adult patients with non-alcoholic fatty liver disease: A meta-analysis study. Hepatol Res 2016;46:862-870. doi:10.1111/hepr.12647

23. Kleiner DE, Brunt EM, Van Natta M, et al. Design and validation of a histological scoring system for nonalcoholic fatty liver disease. Hepatology 2005;41:1313-1321. doi:10.1002/hep.20701

24. Rezaie A, Buresi M, Lembo A, et al. Hydrogen and Methane-Based Breath Testing in Gastrointestinal Disorders: The North American Consensus. Am J Gastroenterol 2017;112:775-784. doi:10.1038/ ajg.2017.46

25. Medeiros JD, Araújo LX, da Silva VL, et al. Characterization of the microbial community in a lotic environment to assess the effect of pollution on nitrifying and potentially pathogenic bacteria. Braz J Biol 2014;74:612-622. doi:10.1590/1519-6984.26712

26. Ryan MC, Wilson AM, Slavin J, Best JD, Jenkins AJ, Desmond PV. Associations Between Liver Histology and Severity of the Metabolic Syndrome in Subjects With Nonalcoholic Fatty Liver Disease. Diabetes Care 2005;28:1222-1224. doi:10.2337/diacare.28.5.1222

27. Stepanova M, Rafiq N, Younossi ZM. Components of metabolic syndrome are independent predictors of mortality in patients with chronic liver disease: a population-based study. Gut 2010;59:1410-1415. doi:10.1136/gut.2010.213553

28. Duarte SMB, Stefano JT, Miele L, et al. Gut microbiome composition in lean patients with NASH is associated with liver damage independent of caloric intake: A prospective pilot study. Nutr Metab Cardiovasc Dis 2018;28:369-384. doi:10.1016/j.numecd.2017.10.014 
29. Boursier J, Mueller O, Barret M, et al. The severity of nonalcoholic fatty liver disease is associated with gut dysbiosis and shift in the metabolic function of the gut microbiota. Hepatology 2016;63:764-775. doi:10.1002/hep.28356

30. Derrien M, Vaughan EE, Plugge CM, de Vos WM. Akkermansia muciniphila gen. nov., sp. nov., a human intestinal mucin-degrading bacterium. Int J Syst Evol Microbiol 2004;54:1469-1476. doi:10.1099/ ijs.0.02873-0

31. Belzer C, VOS W. Microbes inside - from diversity to function: the case of Akkermansia. ISME J 2012;6:1449-1458. doi:10.1038/ismej.2012.6

32. Derrien M, Van Baarlen P, Hooiveld G, Norin E, Müller M, de Vos WM. Modulation of Mucosal Immune Response, Tolerance, and Proliferation in Mice Colonized by the Mucin-Degrader Akkermansia muciniphila. Front Microbiol 2011;2:166. doi:10.3389/fmicb.2011.00166

33. Geerlings S, Kostopoulos I, de Vos WM, Belzer C. Akkermansia muciniphila in the Human Gastrointestinal Tract: When, Where, and How? Microorganisms 2018;6:75. doi:10.3390/microorganisms6030075

34. Png CW, Lindén SK, Gilshenan KS, et al. Mucolytic Bacteria With Increased Prevalence in IBD Mucosa Augment In Vitro Utilization of Mucin by Other Bacteria. Am J Gastroenterol 2010;105:2420-2428. doi:10.1038/ajg.2010.281

35. Swidsinski A, Dörffel Y, Loening-Baucke V, et al. Acute appendicitis is characterized by local invasion with Fusobacterium nucleatum/ necrophorum. Gut 2011;60:34-40. doi:10.1136/gut.2009.191320
36. Greer RL, Dong X, Moraes AC, et al. Akkermansia muciniphila mediates negative effects of IFN $\gamma$ on glucose metabolism. Nat Commun 2016;7:13329. doi:10.1038/ncomms13329

37. Schneeberger M, Everard A, Gómez-Valadés AG, et al. Akkermansia muciniphila inversely correlates with the onset of inflammation, altered adipose tissue metabolism and metabolic disorders during obesity in mice. Sci Rep 2015;5:16643. doi:10.1038/srep16643

38. Everard A, Belzer C, Geurts L, et al. Cross-talk between Akkermansia muciniphila and intestinal epithelium controls diet-induced obesity. Proc Natl Acad Sci U S A 2013;110:9066-9071. doi:10.1073/ pnas. 1219451110

39. Lee JH, Friso S, Choi SW. Epigenetic Mechanisms Underlying the Link between Non-Alcoholic Fatty Liver Diseases and Nutrition. Nutrients 2014;6:3303-3325. doi:10.3390/nu6083303

40. Naito Y, Uchiyama K, Takagi T. A next-generation beneficial microbe: Akkermansia muciniphila. J Clin Biochem Nutr 2018;63:33-35. doi:10.3164/jcbn.18-57

41. Zhai Q, Feng S, Arjan N, Chen W. A next generation probiotic, Akkermansia muciniphila. Crit Rev Food Sci Nutr 2019;59:3227-3236. doi:10.1080/10408398.2018.1517725

42. Plovier H, Everard A, Druart C, et al. A purified membrane protein from Akkermansia muciniphila or the pasteurized bacterium improves metabolism in obese and diabetic mice. Nat Med 2017;23:107-113. doi:10.1038/nm.4236 